\title{
Fructooligosaccharides and mannose affect Clostridium difficile adhesion and biofilm formation in a concentration-dependent manner
}

\author{
Michał Piotrowski ${ }^{1}$ - Dorota Wultańska ${ }^{1}$ • Piotr Obuch-Woszczatyński ${ }^{1} \cdot$ Hanna Pituch $^{1}$
}

Received: 18 June 2019 / Accepted: 9 July 2019 / Published online: 30 July 2019

(C) The Author(s) 2019

\begin{abstract}
The aim of this study was to investigate the effects that prebiotic and candidates for prebiotics on Clostridium difficile strains to adhere to various human epithelial cell lines and to compare the adhesive properties of specific $C$. difficile strains. We also sought to examine the effect of different concentrations of fructooligosaccharides and mannose on the formation of biofilms by C. difficile strains. The influence of cellobiose, fructooligosaccharides, inulin, mannose, and raffinose on the adherence properties of various $C$. difficile strains, including motile 630, non-motile M120, and 10 clinical motile ribotype 027 strains, to non-mucous secreting HT-29, mucous secreting HT-29 MXT, and CCD $841 \mathrm{CoN}$ cells lines. The most effective prebiotics were used in biofilm formation assays. We demonstrated that all $C$. difficile strains adhered to all cell lines. However, the $C$. difficile M120 non-motile strain was statistically more likely to adhere to all three cell lines (CFU median, 40) compared to the motile strains (CFU median, $3 ; p<0.001$ ). Furthermore, among the carbohydrates examined, only fructooligosaccharides and mannose were found to significantly decrease adhesion $(p<0.001)$ of $C$. difficile strains. Alternatively, using a biofilm assay, we observed, via confocal laser scanning microscopy, that sub-inhibitory concentrations (1\%) of fructooligosaccharides and mannose functioned to increase biofilm formation by $C$. difficile. We demonstrated that specific prebiotics and candidate prebiotics exhibit varying anti-adhesive properties towards $C$. difficile in vitro and that treatment with sub-inhibitory concentrations of prebiotics can cause an increase in biofilm formation by $C$. difficile.
\end{abstract}

Keywords Clostridium difficile $\cdot$ Bacterial adhesion $\cdot$ Biofilm formation $\cdot$ Prebiotics, candidate prebiotics

\section{Introduction}

Clostridium difficile (C. difficile) is a Gram-positive anaerobic spore-forming bacterium; it is the primary cause of nosocomial diarrhoea, associated with disturbance of the intestinal microbiota. This microorganism is an etiological agent for antibiotic-associated diarrhoea as well as several clinical complications, including pseudomembranous colitis, toxic megacolon, and intestinal perforation, which has a high mortality rate [1]. The main virulent factors of $C$. difficile are two toxins: toxin A (TcdA; $308 \mathrm{kDa})$ and toxin $\mathrm{B}(\mathrm{TcdB}$; $270 \mathrm{kDa})$. An additional binary toxin-CDT (CDTa, $48 \mathrm{kDa}$ and $\mathrm{CDTb}, 74 \mathrm{kDa}$ ) is produced by some strains $[1,2]$.

Hanna Pituch

hanna.pituch@wum.edu.pl

1 Department of Medical Microbiology, Medical University of Warsaw, Warsaw, Poland
The hypervirulent epidemic strains $\left(\mathrm{A}^{+} \mathrm{B}^{+} \mathrm{CDT}^{+}\right)$are classified as PCR-ribotype 027/North American Pulsotype 1 (NAP1)/restriction endonuclease analysis (REA) BI type (27/NAP1/BI) toxinotype III and are primarily associated with hospital acquired $C$. difficile infections (CDI) [2]. Specifically, PCR-ribotype 027 (RT027) strains are defined as hypervirulent due to their ability to produce high levels of toxins (A and B) together with their high sporulation capacity and antibiotic resistance [2-4]. Thus, patients infected with RT027 are at twice the risk of succumbing to their infection or of developing a severe CDI compared to patients infected with other strains [5]. In a study that examined the causative agents of CDI in hospitals across Poland (2012-2013), RT027 strains were identified as the most prevalent PCR-ribotype [6].

For many pathogens, the capacity to adhere to host tissues is essential for achieving the first stage of pathogenesis. If C. difficile is to successfully colonize the gut, it must first access the epithelial cells, which are coated with a mucus layer. This is accomplished through direct adherence to the mucus [7]. 
Specific cell lines including Caco-2 and HT-29, which have been isolated from colon adenocarcinomas, are commonly employed for in vitro studies examining the attachment of bacterial species. HT-29 cells are characterized as having a small proportion of mucus-secreting cells and columnar absorptive cells, while the mucus-secreting HT29-MTX cells are derived from HT-29 cultures following treatment with methotrexate [8].

Prevention of bacterial adhesion during the early stages of infection can serve to prevent disease development and biofilm formation. Studies have shown that receptor analogs function as efficient anti-adhesive agents and would, thus, be effective primarily against pathogens that bind to human cells. Carbohydrates generally function as efficient cellular receptors and contain similar structures to the glycoproteins or glycolipids for the bacterial adhesins, thereby acting as competitive inhibitors [9]. Prebiotics, which are often saccharides, have thus been described as potential candidates for anti-adhesive therapy. Current definition of a prebiotic is "a substrate that is selectively utilized by host microorganisms conferring a health benefit" [10]. Inulin (INU) and fructooligosaccharides (FOS) are popular oligosaccharide prebiotics that naturally occur in many foods including artichokes, asparagus, leeks, chicory, and garlic [11]. Inulin is a fructan-type polysaccharide carbohydrate, while FOS is a subgroup of inulin, made up of polymers with a degree of polymerization (DP) $\leq 10$. INU and FOS are not digested in the upper gastrointestinal tract and, thus, reach the distal portion of the colon in their full form [12]. Raffinose (RAF) is a trisaccharide made up of galactose, glucose, and fructose, which serves as a functional oligosaccharide, and has applications in medicine and food [13]. Several plants sources such as seeds of soy beans, sugar beets and artichoke (Japanese) are rich in raffinose [13]. Raffinose is considered as candidate prebiotics $[10,13]$. Cellobiose is a disaccharide that is not digested in the human upper gastrointestinal tract. In the presence of human faecal bacteria, cellobiose was observed to significantly increase production of short-chain fatty acids (SCFA) [14]. Dmannose (MAN) is a monosaccharide that has also been reported to exhibit prebiotic activity and may be beneficial for preventing gut dysbiosis by regulating the balance between harmful and commensal bacteria $[15,16]$.

The primary aim of this study was to investigate the effects that specific carbohydrates with prebiotic status and candidates for prebiotics chosen based on their varying degree of polymerization have on the ability of motile and non-motile C. difficile strains to adhere to various human epithelial cell lines and to compare the adhesive properties of specific C. difficile strains to these different cell lines. We also sought to examine the effect of different concentrations of fructooligosaccharides (FOS) and mannose (MAN) on the formation of biofilms by $C$. difficile strains.

\section{Materials and methods}

\section{Preparation of Clostridium difficile cultures and inoculum}

Twelve $C$. difficile strains were used in this study. Among them were 2 control strains, $C$. difficile 630 characterized as ribotype 012 (RT012) and an epidemic strain isolated in 1985 from Zurich, Switzerland, C. difficile M120 (RT078), and 10 tested strains that were all clinical isolates and toxigenic belonging to the PCR-ribotype (RT027). These strains were isolated from symptomatic patients across Poland [17, 18]. All C. difficile strains were collected in the Anaerobic Laboratory, in the Department of Medical Microbiology, at the Medical University of Warsaw. C. difficile strains were stored at $70{ }^{\circ} \mathrm{C}$ in a Microbank ${ }^{\mathrm{TM}}$ bacterial storage system (Pro-Lab Diagnostics, UK). The strains were thawed before use in experiments, cultured on Columbia Agar plates with 5\% sheep blood (Beckton Dickinson, USA), and incubated at $37^{\circ} \mathrm{C}$ for $48 \mathrm{~h}$ under anaerobic conditions. Clinical isolates were confirmed as $C$. difficile via mass spectrometry (Vitek MS bioMérieux, France). RTs were determined using methods as described by Stubbs et al. [19]. Inoculums were prepared with suspension colonies of $C$. difficile cultured at $37^{\circ} \mathrm{C}$ for $48 \mathrm{~h}$ under anaerobic conditions on Columbia Agar with 5\% sheep blood (bioMérieux, France) and adjusted to a 3.0 McFarland standard.

\section{Motility assay}

Motility assays were performed using motility agar tubes containing brain heart infusion (BHI; Difco, USA) medium $(0.175 \%$ agar) [20]. The media was inoculated by stabbing with one colony of $C$. difficile that had been previously cultured on Columbia Agar with 5\% sheep blood. Results from clinical isolates were compared to those of control strains.

\section{Prebiotics preparation}

Cellobiose (CEL), fructooligosaccharides (FOS), inulin (INU) (from chicory), mannose (MAN), and raffinose (RAF) were purchased from Sigma-Aldrich (USA). Stock solutions (10\% w/v) were prepared using deionized sterile water, microfiltered using a syringe filter $\left(0.2 \mu \mathrm{m}\right.$, Corning, USA), and stored at $4{ }^{\circ} \mathrm{C}$.

\section{Cell cultures}

Three human epithelial cell lines were employed throughout the study, namely, HT-29 which are phenotypically nonmucus-secreting cells and were passaged 15-25 prior to use (from the cell-line library at the Anaerobe Laboratory, Department of Medical Microbiology), mucus-secreting HT29 MTX, passaged 5-15 times (European Collection of 
Authenticated Cell Cultures, ECACC, UK) and Homo sapiens normal colon CCD $841 \mathrm{CoN}$ cells, passaged 5-15 times (American Type Culture Collection, ATCC, USA). Cells were stored in liquid nitrogen at $-196{ }^{\circ} \mathrm{C}$. Cells were cultured in $25 \mathrm{mM}$ 4-(2-hydroxyethyl)-1-piperazineethanesulfonic acid (HEPES) Dulbecco's modified Eagle medium (DMEM; Life Technologies, UK) with high glucose (4.5 g/L D-glucose), Lglutamine $(4.0 \mathrm{mM})$, supplemented with $10 \%$ heat inactivated (30 $\mathrm{min}$ at $56{ }^{\circ} \mathrm{C}$ ) fetal bovine serum (FBS) (Thermo Scientific, USA), and $1 \%$ antibacterial solution (Life Technologies, USA) containing 10,000 $\mu \mathrm{g} / \mathrm{mL}$ streptomycin, $10,000 \mathrm{U} / \mathrm{mL}$ penicillin, and $0.1 \%$ amphotericin $\mathrm{B}(250 \mu \mathrm{g} /$ $\mathrm{mL}$ ). All cells were maintained in $75-\mathrm{cm}^{2}$ flasks (Corning, USA) and incubated at $37{ }^{\circ} \mathrm{C}$ with $5 \% \mathrm{CO}_{2}$ and $95 \%$ relative humidity. Media was changed every two days. After reaching $100 \%$ confluence, cells (the surface is completely covered by the cells) were washed with $10 \mathrm{~mL}$ warm $\left(37^{\circ} \mathrm{C}\right)$ phosphate buffered saline (PBS; Thermo Fisher, USA) and harvested with $3 \mathrm{~mL}$ of $0.25 \%$ trypsin-ethylenediaminetetraacetic acid (EDTA; Sigma-Aldrich, USA) for $5 \mathrm{~min}$ at $37^{\circ} \mathrm{C}$. Trypsin was deactivated by adding $10 \mathrm{~mL}$ of fresh DMEM with $10 \% \mathrm{FBS}$, followed by centrifugation at $1500 \times g$ for $5 \mathrm{~min}$. Supernatant was discarded, and the pelleted cells were resuspended in $1 \mathrm{~mL}$ of fresh DMEM. Cell counts was performed using a Thoma chamber and seeded onto 24-well plates (Corning, USA) at a concentration of $10^{4}$ cells per well, or re-cultured in a new sterile flask. Cells were observed daily and examined for growth and contamination using an inverted microscope (PZO, Poland). Media without antibiotics and antimycotic substances was used for the last media change. Experiments were performed on mature cells, which was 15 days after seeding HT-29 and CCD $841 \mathrm{CoN}$ cells and 21 days after seeding HT-29 MXT cells [21, 22].

\section{Adhesion of $C$. difficile strains to human epithelial cell lines}

The method employed for determining adhesive properties of C. difficile was described previously by Altamimi et al. with specific modifications [22]. All cell lines were prepared as described above. After reaching sub-confluence (70\%-80\%), cells in 24-well plates were washed twice with PBS, and $400 \mu \mathrm{L}$ of fresh pre-warmed $\left(37{ }^{\circ} \mathrm{C}\right)$ DMEM without antibiotic/antimycotic solution and with a $1 \%$ final saccharide concentration was then added. Medium without saccharides was employed as a negative control. Prepared plates were incubated for $4 \mathrm{~h}$ under the abovementioned cell culture conditions. Afterwards, $100 \mu \mathrm{L}$ of bacterial inoculum was prepared and added to each well and incubated for $1 \mathrm{~h}$. Medium was then aspirated, and wells were washed twice with PBS. The cells were trypsinized for $10 \mathrm{~min}$ at $37^{\circ} \mathrm{C}$ and $500 \mu \mathrm{L}$ of fresh media with $10 \%$ FBS was added to deactivate the trypsin. The contents of each well were transferred to sterile
Eppendorf tubes and diluted 10 times using PBS, and $20 \mu \mathrm{L}$ was then used to inoculate Columbia Agar with 5\% sheep blood. The plates were incubated for $48 \mathrm{~h}$ at $37{ }^{\circ} \mathrm{C}$ under anaerobic conditions. Every dilution was seeded in duplicate and each assay was performed in triplicate. Colonies were counted and averaged, adhesion percentage was calculated using the formula below (where control represented $100 \%$ adhesion) [22].

Adhesion $(\%)=\frac{\text { bacterial count sample }}{\text { bacterial count control }} \times 100$

\section{Biofilm growth and influence of prebiotics on biofilm formation}

The most anti-adhesively effective prebiotics FOS and MAN were used in biofilm formation assays. BHI media with different concentrations of saccharides/prebiotics $(1 \%, 2 \%, 4 \%$, $8 \%$ ) was pipetted into each well of 96 -well flat-bottom microplates (Nunc, Denmark). Three wells for each strain were subsequently inoculated with $20 \mu \mathrm{L}$ of $C$. difficile culture. Wells containing BHI broth without inoculum were used as negative controls, while positive controls consisted of inoculated wells without prebiotic treatment. Plates were incubated at $37^{\circ} \mathrm{C}$ for $48 \mathrm{~h}$ under anaerobic conditions. After $48 \mathrm{~h}$, the liquid phase of each well was aspirated using sterile pipettes, washed twice with PBS to remove unattached cells, and air dried at $37^{\circ} \mathrm{C}$ for $15 \mathrm{~min}$. Each well was then stained with $1 \%$ crystal violet (CV; Chempur, Poland) for $10 \mathrm{~min}$. The $\mathrm{CV}$ was removed, wells were washed 8 times with PBS, and air dried for $15 \mathrm{~min}$ at $37^{\circ} \mathrm{C}$. The stain was dissolved with $96 \%$ ethanol (Hurt-Chem, Poland), and absorbance was measured at $\mathrm{A}_{620}$ (Bio-Rad 550 Microplate Reader, Bio-Rad, USA).

\section{Confocal laser scanning microscopy}

Specimens were visualized via confocal laser scanning microscopy (CLSM) according to methods previously described by Waack et al. with modifications [23]. Biofilms were grown on sterile 10-mm-diameter glass bottom dishes (Nunc, Denmark). Overnight cultures of $C$. difficile were diluted in fresh BHI with or without prebiotics. To the experimental conditions, $1 \%$ and $8 \%$ concentrations of FOS and MAN were added. At $1 \%$, FOS and MAN were seen to induce biofilm growth; while at $8 \%$, both prebiotics effectively inhibited C. difficile biofilm formation. Biofilms were allowed to grow for $48 \mathrm{~h}$ at $37^{\circ} \mathrm{C}$ under anaerobic conditions. Mature biofilms were washed twice using $10 \mathrm{mM} \mathrm{MgSO}_{4}$. Biofilms were then stained with acridine orange $(10 \mu \mathrm{g} / \mathrm{mL})$ for $30 \mathrm{~min}$ in the dark. Dishes were washed twice with $10 \mathrm{mM} \mathrm{MgSO}_{4}$. Imaging was performed using a Nikon A1R MP microscope with a Nikon Ti Eclipse series (Nikon, Japan) under $\times 60$ 
objective lens using immersion oil. Images were acquired at $2040 \times 2048$ pixels using a Z-step of $0.1 \mu \mathrm{m}$. Acridine orange was detected using an excitation wavelength of $488 \mathrm{~nm}$ and emission wavelength of 500-550 nm. Images were processed and analysed with NIS-Elements AR v. 4.10 software.

\section{Statistical analysis}

Statistical analysis was performed using Statistica software (version 13, StatSoft, Poland). Normal distribution of values was confirmed using Shapiro-Wilk test. Differences in adhesion between motile and non-motile strains were evaluated by Mann-Whitney $U$ test. The effect of prebiotics on $C$. difficile adhesion was calculated using Kruskal-Wallis one-way analysis of variance followed by Dunn's test for comparison. Differences in biofilm formation were calculated via oneway analysis of variance (ANOVA) followed by Tukey's post-hoc test.

\section{Results}

Our study assessed the adhesive capacity of motile and nonmotile $C$. difficile, the effects of 5 prebiotics on this adhesive effect, and the influence of chosen prebiotics (FOS and MAN) on biofilm formation.

\section{C. difficile adhesion to human epithelial cell lines}

We compared adhesive properties of 10 strains of $C$. difficile RT027 to three human epithelial cell lines, without prebiotics. Our results showed that the specific cell line did not significantly affect adhesion $(p=0.65)$, with a median CFU of 5.5 for HT-29 and HT-29 MXT and 4.5 for CCD $841 \mathrm{CoN}$ cells (Fig. 1).

\section{Influence of motility on adhesive properties of $C$. difficile}

We next compared the adhesive properties of different $C$. difficile strains with different motility properties. These strains included a motile $C$. difficile 630 strain, a non-motile C. difficile M120 strain, and 10 motile clinical RT207 strains. The number of CFUs was compared to those of control groups. We observed a strong, statistically significant correlation $(p<0.001)$ between the number of CFUs and strain motility. The non-motile strain was found to be more likely to adhere (CFU median, 40) to all three cell lines compared to the motile 630 strain (CFU median, 3) or the clinical RT027 strains (CFU median, 5). These relationships are presented in Fig. 2.

\section{Effect of prebiotics on adhesive properties of $C$. difficile}

The primary aim of this study was to examine the effect of 5 prebiotics on the adhesion of $12 C$. difficile strains (two control strains and 10 clinical strains) to three human epithelial cell lines. FOS and MAN were found to significantly interfere with efficient bacterial adhesion in all cell lines $(p<0.001)$, while RAF only interfered with the adhesive properties of $C$. difficile to the non-mucus-secreting HT-29 cell line ( $p=$ 0.008). Moreover, CEL and INU were determined to be less
Fig. 1 Median adhesion of $C$. difficile strains to the three cell lines

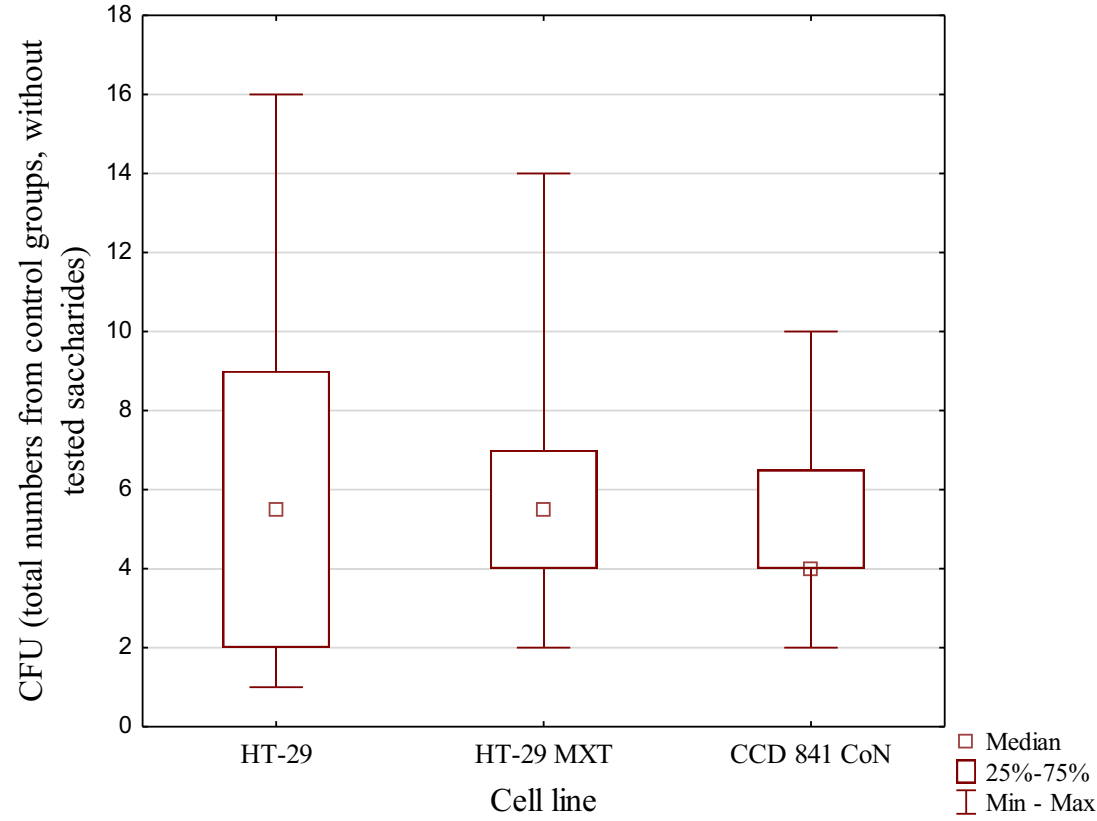


Fig. 2 Adhesion of motile clinical C. difficile strains belonging to RT027 and two control strains non-motile M120 and motile 630

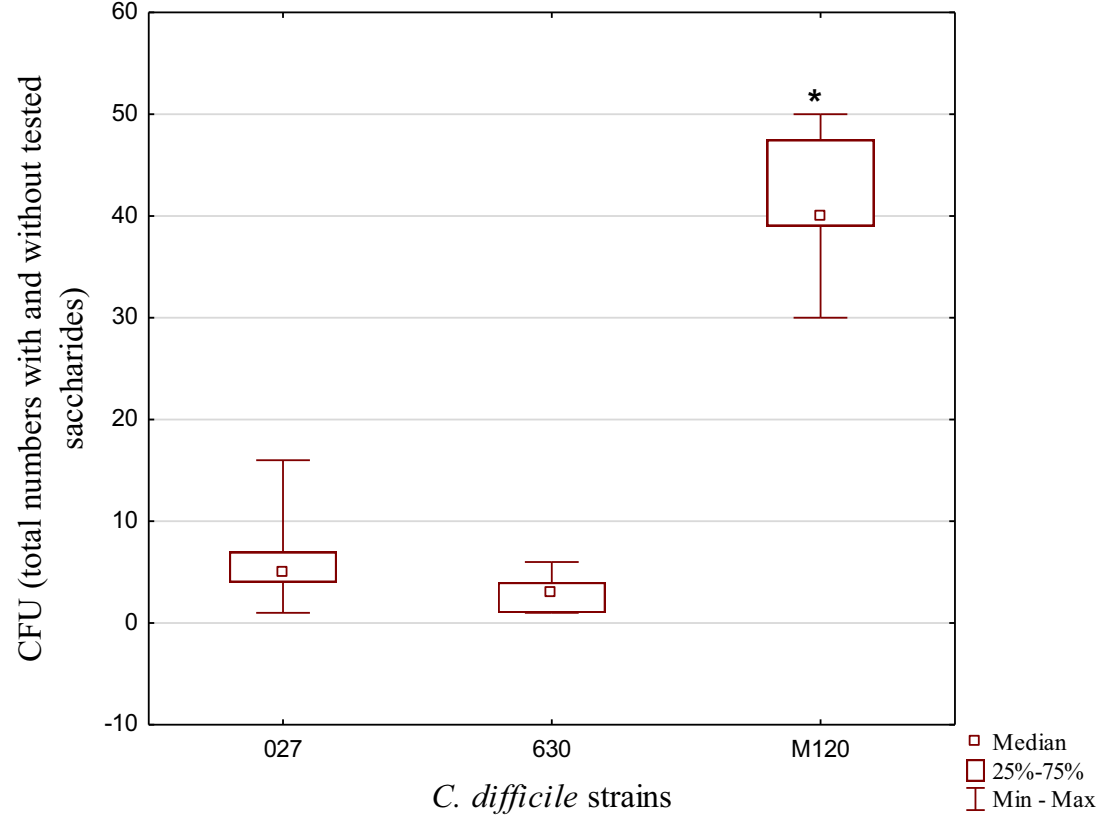

effective at inhibiting $C$. difficile adhesion in all cell lines $(p>0.05)$ (Table 1 and Fig. 3).

\section{Influence of FOS and MAN on C. difficile biofilm formation}

To determine the effect of prebiotics on biofilm formation, we only included those that elicited consistent inhibitory effects on the adhesion of $C$. difficile in the previous experiment, namely FOS and MAN. All 12 strains of bacteria were included in this study. Our results revealed that the $C$. difficile M120 strain possessed the strongest ability to form biofilm in vitro with a mean absorbance $(\mathrm{A} 620 \mathrm{~nm})$ from three measurements of 1.93. Among the clinical strains, the highest amount of biofilm was produced by strains 2 (mean A620, 0.80), 8 (mean A620, 0.77), 6 (mean A620, 0.75), and 9 (mean A620, 0.70). Alternatively, the least amount of biofilm was produced by strain 5 (mean A620, 0.33).

We examined the effects of the prebiotics at concentrations of $1 \%, 2 \%, 4 \%$, and $8 \%$. At $1 \%$, both MAN and FOS acted to induce biofilm growth for all $C$. difficile strains. However, statistically significant differences were observed for strain 630 (MAN 1\% $p<0.001$; FOS $1 \%$ $p<0.001)$. In addition, treatment with $2 \%$ FOS was found to induce biofilm growth of $C$. difficile $630(p=0.014)$. However, all other treatments with prebiotics added at concentrations of $2 \%$ and $4 \%$ did not significantly affect biofilm formation. When added at a concentration of $8 \%$, MAN acted to significantly reduce biofilm formation by $C$. difficile $630(p=0.02)$ and M120 $(p=0.004)$. Similar observations were made for $8 \%$ FOS with these two strains of bacteria ( $p=0.025$ and $p=0.028$, respectively).
However, treatment of clinical RT027 strains with MAN and FOS did not cause significant differences in biofilm formation. However, inhibition were observed in strain no 2 following treatment with $8 \%$ MAN $(p=0.30)$ and $8 \%$ FOS $(p=0.22)$, in strain no 7 by $8 \% \operatorname{MAN}(p=0.18)$ and $8 \%$ FOS $(p=0.14)$, in strain no 8 by $8 \%$ MAN $(p=0.24)$ and $8 \%$ FOS $(p=0.34)$, and in strain no 4 by $8 \%$ FOS $(p=0.18)$. We, therefore, categorized prebiotic concentrations of $8 \%$ as inhibitory, and lower concentrations (1\%, $2 \%, 4 \%$ ) as sub-inhibitory (Fig. 4).

\section{Confocal laser scanning microscopy}

To visualize the effects of FOS and MAN, two concentrations of these substances were utilized in culture with 3 different C. difficile strains, namely, 630, M120, and one clinical C. difficile RT027 that has previously been shown to produce the highest level of biofilm on titration plates. Prebiotics were used at an $8 \%$ concentration, which demonstrated strong inhibitory effects on biofilm formation by $C$. difficile, and at a sub-inhibitory $(1 \%)$ concentration which was found to induce the process of biofilm formation. Images from confocal microscopy (Fig. 5.) confirmed the results from the experiment with titration plates. $C$. difficile 630 strain was found to form a thin biofilm layer in the control without prebiotics (Fig. 5. 630 A), and the other strains, M120 and RT027, produced higher amounts of biofilm in the control. The sub-inhibitory concentration (1\%) of FOS and MAN (Fig. 5 B, D) acted to increase the density and roughness of $C$. difficile biofilm, most notably within strain 630 cultures. Further, the biofilm produced by strain M120 in culture with $1 \%$ FOS or MAN became rugged with holes and elongated forms (Fig. 5, M120 B and D). 


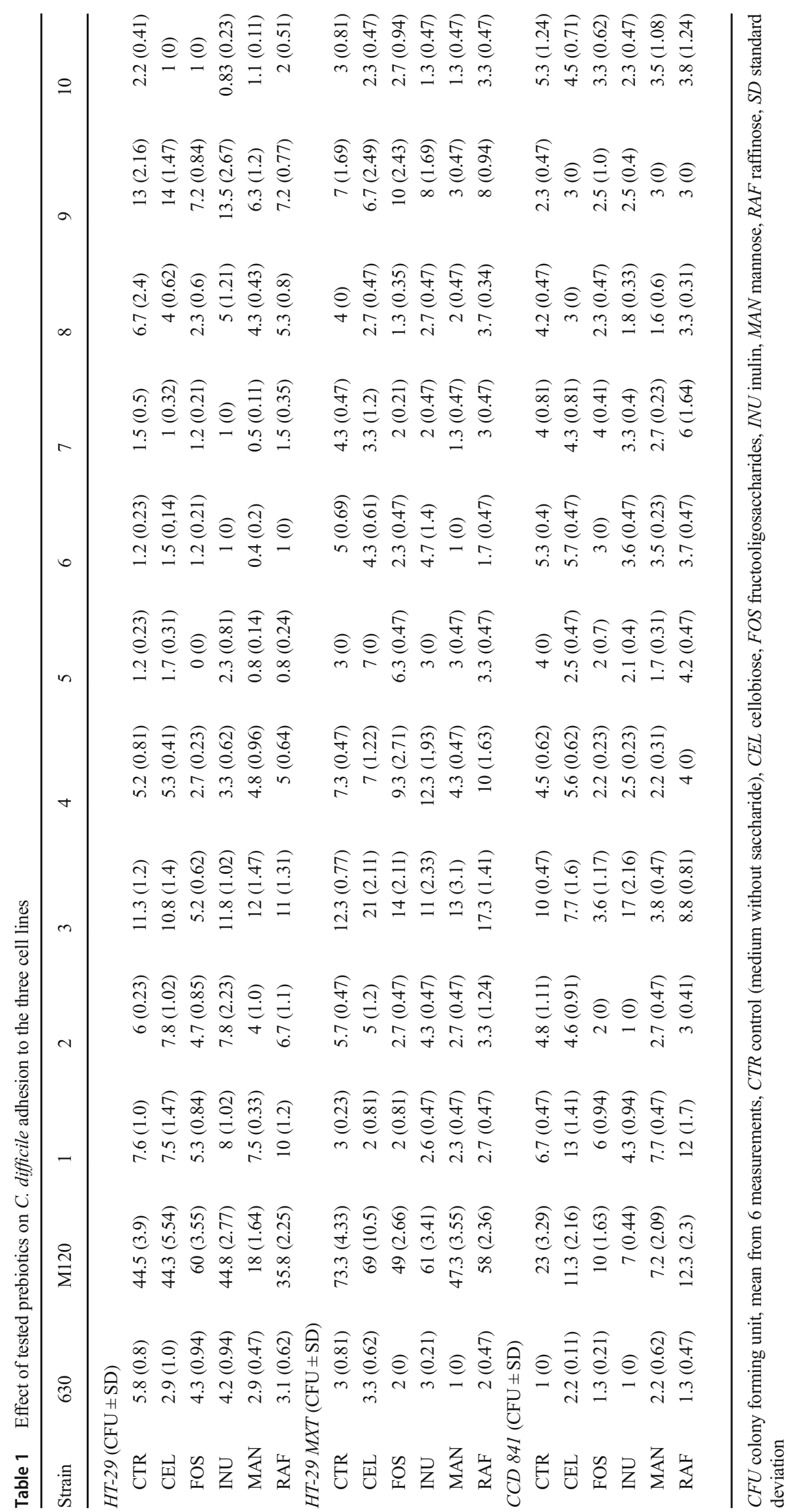



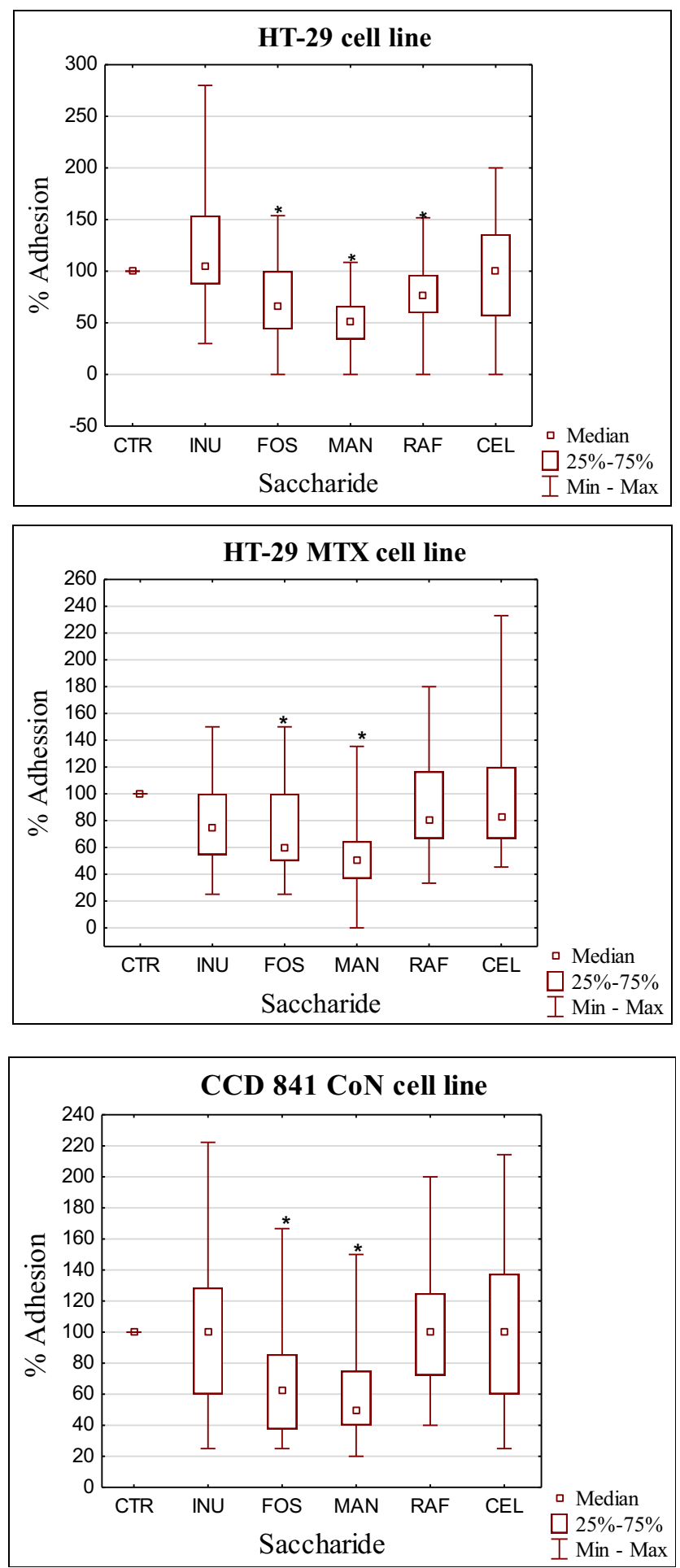

Fig. 3 Effect of examined prebiotics on adhesion of $C$. difficile to three cell lines

Alternatively, higher concentrations (8\%) of prebiotics resulted in decreased biofilm biomass production with a thinner layer and a smaller number of bacterial aggregates in all tested strains compared to the control (Fig. $5 \mathrm{C}$ and E).

\section{Discussion}

C. difficile is the most common etiological factor for nosocomial diarrhoea. The increase in CDI incidence has been caused by emergence of hyperepidemic strains, especially those belonging to the genotype NAP1/B1/RT027. These strains often exhibit multi-drug resistance and highly expressed virulence factors, such as higher toxin production and high spore resistance to adverse conditions [6]. Furthermore, a study performed by Collins et al. reported that treatment with the disaccharide trehalose acted to enhance pathogenic $C$. difficile virulence [24].

In the current study, we examined the effects of specific carbohydrates prebiotics and candidate prebiotics: CEL, FOS, INU, MAN, and RAF on various $C$. difficile strains. We focused primarily on clinical strains characterized as PCRribotype 027 due to their hyper-pathogenic characteristics. We, specifically, investigated how non-digestible prebiotics affect in vitro adhesion of $C$. difficile, which is the initial step of colonization and biofilm formation.

FOS and MAN exhibited the strongest anti-adhesion potential in all the 3 human epithelial cell lines. Hartman et al. reported on the anti-adhesive potential of mannosides on $E$. coli. They found that mannose and mannans decreased $E$. coli adhesion to HT-29 cells by up to $90 \%$ [25]. Further, Shoaf et al. employed FOS to reduce adherence of enteropathogenic E. coli (EPEC) to Caco-2 cells and reported approximately $40 \%$ inhibition of adherence [26]. Altamimi et al. also investigated the effect of different oligosaccharides, including raffinose and cellobiose on gut bacteria, including $C$. difficile. However, no significant effects were observed in this study. Other carbohydrates (chitooligose, lactulose, stachyose) have also not exhibited significant anti-adhesive properties in HT-29 cell lines (non-mucus and mucus secreting) [22]. Similarly, in our study, CEL and INU demonstrated the largest CFU median in all the 3 cell lines and promoted adhesion in only a few bacterial strains (data not shown), while RAF was found to only significantly inhibit adhesion of $C$. difficile in HT-29 cells. Alternatively, Wang et al. reported on the effect of carbohydrates on adhesion of intestinal pathogens to HT-29 cells. Mannose was found to reduce adhesion of Vibrio cholerae by $60 \%$ and Campylobacter jejuni by $30 \%$. Moreover, FOS inhibited adhesion of Salmonella Typhimurium by $71.4 \%$. However, these saccharides did not effectively inhibit adhesion of $E$. coli [27]. The mechanisms responsible for inhibiting and promoting adhesion are not clearly understood. However, sugars have been shown to function as adhesion inhibitors and affect the expression of surface proteins and adhesins of bacteria. Hence, further examination of these mechanisms will be carried out in the future.

Our results suggest that specific prebiotics can affect the formation of biofilms by certain $C$. difficile strains. Media supplemented with $8 \%$ FOS and MAN functioned to statistically significantly decrease biofilm formation by $C$. difficile 630 and C. difficile M120. However, in the clinical strains, significantly 

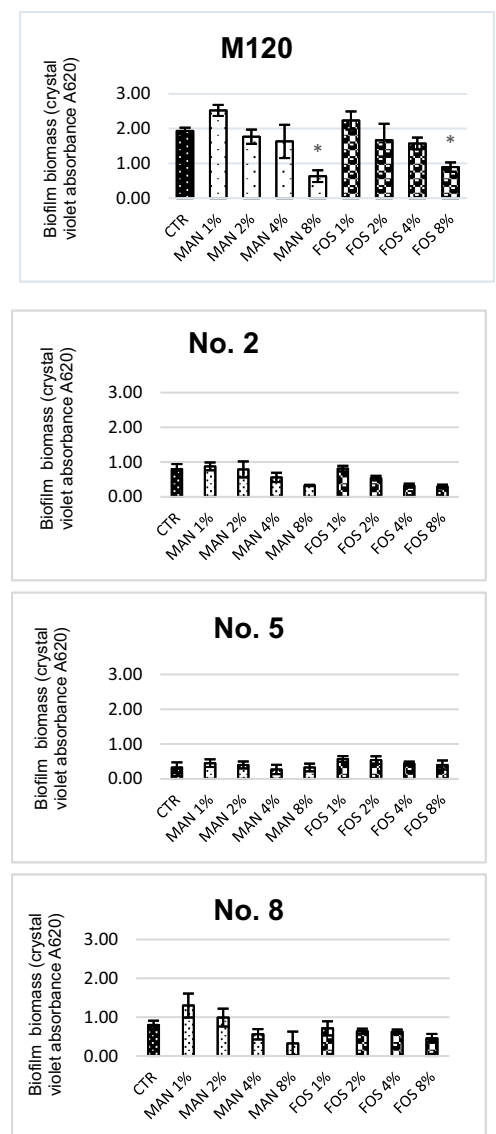
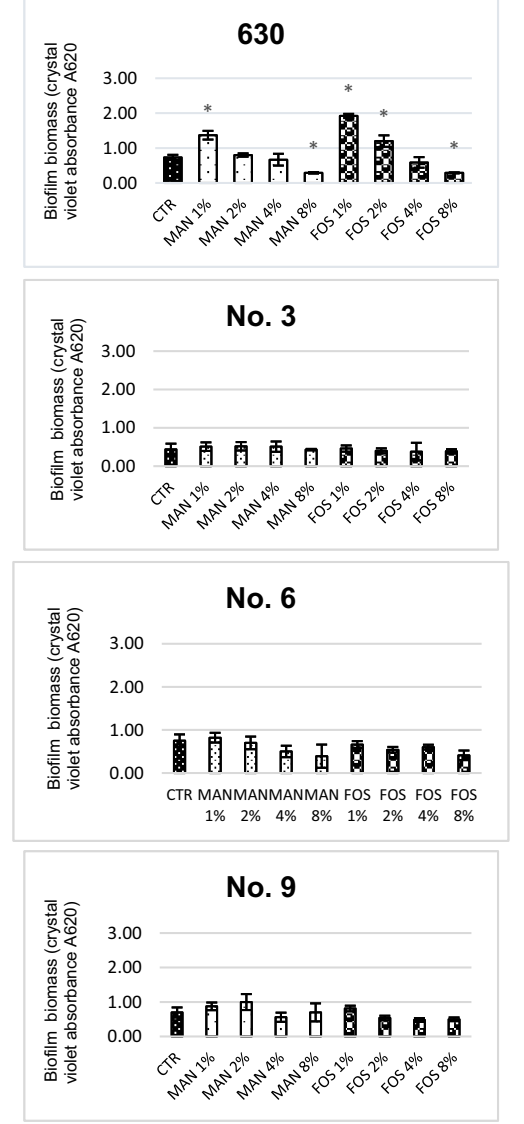

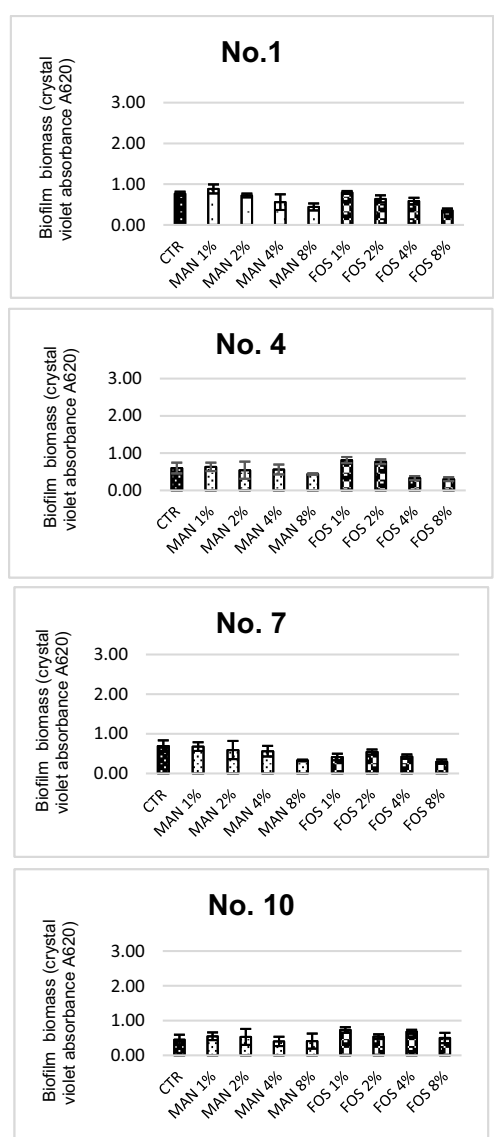

Fig. 4 Average biofilm formation by examined C. difficile strains with different concentrations FOS and MAN

decreased biofilm formation not observed. Interestingly, we also observed that biofilm formation was enhanced in the presence of low concentrations of prebiotics, most notably with $1 \%$ (sub-inhibitory) FOS and MAN. However, for RT027 clinical strains, observations were not statistically significant. Images from confocal laser scanning microscopy confirmed these results and allowed us to visualize the 3D architecture of biofilms. At sub-inhibitory concentration (1\%) of FOS and MAN, an increase in roughness and changes in homogeneity were observed, resulting in changes from a smooth biofilm surface to a more heterologous surface and a 3D architecture containing many aggregates. The biofilm formed by $C$. difficile 630, in the absence of prebiotics, was the thinnest of the three tested strains which corroborated with results from Semenyuk et al. who described $C$. difficile 630 as a weak biofilm producer [28]. Additionally, our results showed increased biofilm formation by $C$. difficile following exposure to a sub-inhibitory concentration (1\%) of FOS and MAN. Similarly, Creti et al. described strong biofilm production by wild-type Enterococcus faecalis after incubation with $1 \%$ MAN and fructose [29]. These results suggest that the presence of oligosaccharides in food may influence colonization and biofilm formation by bacteria in the human gastrointestinal tract.
To our knowledge, this is first study to investigate the effects of prebiotics on biofilm formation by $C$. difficile and, thus, requires further investigation. Powell et al. examined the effects of alginate oligosaccharide on Pseudomonas aeruginosa biofilm and microscopy imaging demonstrated a dose-depending reduction on biofilm formation. Moreover, alginates at $2 \%$ and $6 \%$ concentrations functioned to decrease the thickness of $P$. aeruginosa biofilm [30].

Many studies examining the adhesive properties of microorganisms employ epithelial cell lines such as HT-29 or Caco-2. We, however, used an additional mucussecreting epithelial cell line, namely HT-29 MXT and epithelial cells from healthy human CCD $841 \mathrm{CoN}$ for comparison. However, we observed no significant differences in adhesion of $C$. difficile RT027 to these 3 cell lines. These results were not in agreement with those reported by Altamimi et al. This study revealed that $C$. difficile preferred mucus secreting epithelial cells over nonmucus-secreting ones; however, only one reference strain ATCC 43255 was used in these assays [22].

Although no significant differences were observed in adherence of the clinical strains to the different cell lines, we did determine that the non-motile $C$. difficile M120 

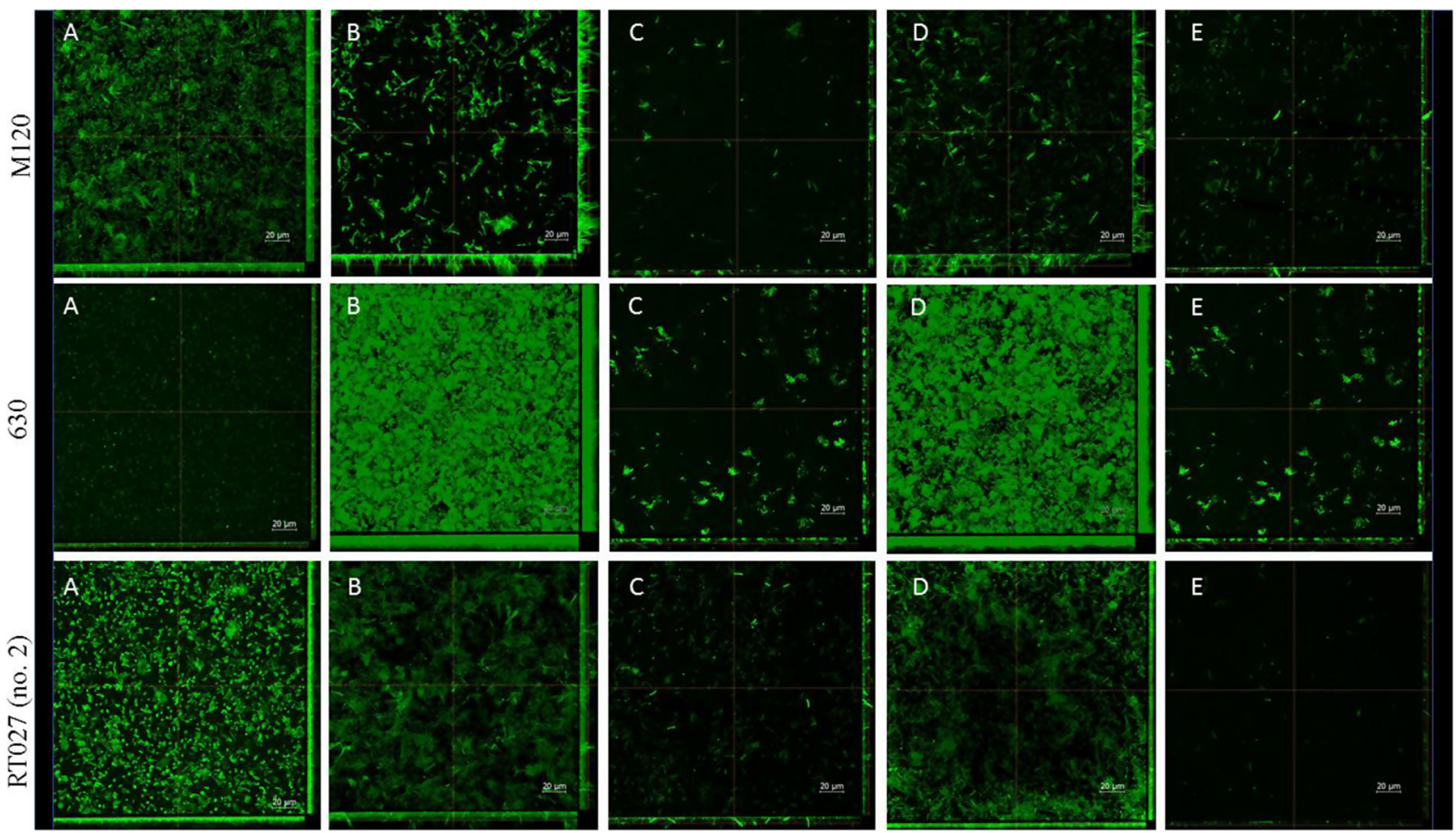

Fig. 5 Effects of FOS and MAN on C. difficile biofilm formation. Representative confocal microscopy images of horizontal (xy) and vertical (xz and yz) projections of $C$. difficile biofilm structures. Vertical view was obtained using maximum intensity projection as a crosssection through marked line

strain adhered more strongly than the motile $C$. difficile 630 or RT027 strains. The M120 strain has deletion of the entire F3 region which is responsible for encoding flagellin [31]. However, mice experiments employing the 630 strain with other flagellar mutants revealed that flagella are not required for adherence and colonization by this $C$. difficile strain [32]. Thus, higher adhesion of nonmotile strains may be simply explained by more rapid settling of the bacterial cells onto the epithelia compared to that by motile strains.

\section{Conclusion}

Specific prebiotics exhibit anti-adhesive properties and are safe and ecologically friendly. FOS and MAN possessed the highest anti-adhesive activity against $C$. difficile strains, thereby leading to decreased biofilm formation. For clinical strains, the effect was observable; however, values were not statistically significant. Importantly, we also determined that sub-inhibitory concentrations of FOS and MAN serve to enhance biofilm formation, which is better visualized using confocal microscopy imaging than crystal violet staining. To our knowledge, this is the first report to address the effects of prebiotics and candidate prebiotics on adhesion and biofilm formation of hypervirulent RT027 C. difficile strains.
Acknowledgements The authors would like to thank Prof. Brendan Wren, Department of Pathogen Molecular Biology, London School of Hygiene and Tropical Medicine, UK, for kindly providing C. difficile 630 and M120 control strains. We would like to thank Dr. Bohdan Paterczyk, Faculty of Biology, University of Warsaw, for the help in CLSM imaging. We wish to thank the Polish clinical microbiologist for the $C$. difficile strains.

Funding This work was supported by the National Science Centre in Cracow, Poland (Grant number: UMO:2017/25/N/NZ6/01763).

\section{Compliance with ethical standards}

Conflict of interest The authors report no conflicts of interest.

Ethical approval Clinical C. difficile strains were collected as part of routine hospitals surveillance. Ethical approval and informed consent were not required.

Open Access This article is distributed under the terms of the Creative Commons Attribution 4.0 International License (http:// creativecommons.org/licenses/by/4.0/), which permits unrestricted use, distribution, and reproduction in any medium, provided you give appropriate credit to the original author(s) and the source, provide a link to the Creative Commons license, and indicate if changes were made.

\section{References}

1. Rupnik M, Wilcox MH, Gerding DN (2009) Clostridium difficile infection: new developments in epidemiology and pathogenesis. Nat Rev Microbiol 7:526-536. https://doi.org/10.1038/ nrmicro2164 
2. Cohen MB (2009) Clostridium difficile infections: emerging epidemiology and new treatments. J Pediatr Gastroenterol Nutr 48:6365. https://doi.org/10.1097/MPG.0b013e3181a118c6

3. Kenters N, Huijskens EGW, de Wit SCJ et al (2017) Effectiveness of various cleaning and disinfectant products on Clostridium difficile spores of PCR ribotypes 010, 014 and 027. Antimicrob Resist Infect Control 6:54. https://doi.org/10.1186/s13756-0170210-3.

4. Freeman J, Vernon J, Morris K et al (2015) Pan-European longitudinal surveillance of antibiotic resistance among prevalent Clostridium difficile ribotypes. Clin Microbiol Infect 21:248.e9248.e16. https://doi.org/10.1016/j.cmi.2014.09.017

5. Miller M, Gravel D, Mulvey M et al (2010) Health care-associated Clostridium difficile infection in Canada: patient age and infecting strain type are highly predictive of severe outcome and mortality. Clin Infect Dis 50:194-201. https://doi.org/10.1086/649213

6. Pituch H, Obuch-Woszczatyński P, Lachowicz D et al (2015) Hospital-based Clostridium difficile infection surveillance reveals high proportions of PCR ribotypes 027 and 176 in different areas of Poland, 2011 to 2013. Euro Surveill 20. https://doi.org/10.2807/ 1560-7917.ES.2015.20.38.30025

7. Karjalainen T, Barc MC, Collignon A et al (1994) Cloning of a genetic determinant from Clostridium difficile involved in adherence to tissue culture cells and mucus) Infect Immun 62: $4347-4355$

8. Lesuffleur T, Barbat A, Dussaulx E, Zweibaum A (1990) Growth adaptation to methotrexate of HT-29 human colon carcinoma cells is associated with their ability to differentiate into columnar absorptive and mucus-secreting cells. Cancer Res 50:6334-6343

9. Ofek I, Hasty DL, Sharon N (2003) Anti-adhesion therapy of bacterial diseases: prospects and problems. FEMS Immunol Med Microbiol 38:181-191. https://doi.org/10.1016/S0928-8244(03) 00228-1

10. Gibson GR, Hutkins R, Sanders ME et al (2017) Expert consensus document: The International Scientific Association for Probiotics and Prebiotics (ISAPP) consensus statement on the definition and scope of prebiotics. Nat Rev Gastroenterol Hepatol 14:491-502. https://doi.org/10.1038/nrgastro.2017.75

11. Van Loo J, Coussement P, De Leenheer L et al (1995) On the presence of inulin, oligofructose as natural ingredients in the Western diet. Crit Rev Food Sci Nutr 35:525-552

12. Niness KR (1999) Inulin and oligofructose: what are they? J Nutr 129(7 Suppl):1402S-1406S. https://doi.org/10.1093/jn/129.7. $1402 \mathrm{~S}$

13. Adamberg K, Adamberg S, Ernits K et al (2018) Composition and metabolism of fecal microbiota from normal and overweight children are differentially affected by melibiose, raffinose and raffinose-derived fructans. Anaerobe. 52:100-110. https://doi.org/ 10.1016/j.anaerobe.2018.06.009

14. van Zanten GC, Krych L, Röytiö H et al (2014) Synbiotic Lactobacillus acidophilus NCFM and cellobiose does not affect human gut bacterial diversity but increases abundance of lactobacilli, bifidobacteria and branched-chain fatty acids: a randomized, double-blinded cross-over trial. FEMS Microbiol Ecol 90:225-236. https://doi.org/10.1111/1574-6941.12397

15. Sharma V, Smolin J, Nayak J et al (2018) Mannose alters gut microbiome, prevents diet-induced obesity, and improves host metabolism. Cell Rep 24:3087-3098. https://doi.org/10.1016/j.celrep. 2018.08.064

16. Sundu B, Kumar A, Dingle J (2006) Palm kernel meal in broiler diets: effect on chicken performance and health. World Poultry Sci J 62:316-325. https://doi.org/10.1079/WPS2005100

17. Roberts AP, Smits WK (2018) The evolving epidemic of Clostridium difficile 630. Anaerobe 53:2-4. https://doi.org/10. 1016/j.anaerobe.2018.04.015
18. Kumar A, Davenport KW, Vuyisich G et al (2018) Complete genome sequences of historic Clostridioides difficile food-dwelling Ribotype 078 strains in Canada identical to that of the historic human clinical strain M120 in the United Kingdom. Microbiol Resour Announc 7:pii: e00853-18. https://oi.org/10.1128/MRA. 00853-18

19. Stubbs D, Brazier JS, O'Neill JL, Duerden BI (1999) PCR targeted to the 16S-23S rRNA gene intergenic spacer region of Clostridium difficile and construction of a library consisting of 116 different PCR ribotypes. J Clin Microbiol 37:461-463

20. Twine SM, Reid CW, Aubry A et al (2009) Motility and flagellar glycosylation in Clostridium difficile. J Bacteriol 191:7050-7062. https://doi.org/10.1128/JB.00861-09

21. Pantosti A, Cerquetti M, Colangeli R, D’Ambrosio F (1994) Detection of intestinal and extra-intestinal strains of enterotoxigenic Bacteroides fragilis by the HT-29 cytotoxicity assay. J Med Microbiol 41:191-196

22. Altamimi M, Abdelhay O, Rastall RA (2016) Effect of oligosaccharides on the adhesion of gut bacteria to human HT-29 cells. Anaerobe 39:136-142. https://doi.org/10.1016/j.anaerobe.2016. 03.010

23. Waack U, Nicholson TL (2018) Subinhibitory concentrations of amoxicillin, lincomycin, and oxytetracycline commonly used to treat swine increase Streptococcus suis biofilm formation. Front Microbiol 9:2707. https://doi.org/10.3389/fmicb.2018.02707

24. Collins J, Robinson C, Danhof H et al (2018) Dietary trehalose enhances virulence of epidemic Clostridium difficile. Nature 553(7688):291-294. https://doi.org/10.1038/nature25178

25. Hartmann M, Papavlassopoulos H, Chandrasekaran V et al (2012) Inhibition of bacterial adhesion to live human cells: activity and cytotoxicity of synthetic mannosides. FEBS Lett 586:1459-1465. https://doi.org/10.1016/j.febslet.2012.03.059

26. Shoaf K, Mulvey GL, Armstrong GD, Hutkins RW (2006) Prebiotic galactooligosaccharides reduce adherence of entheropathogenic Escherichia coli to tissue culture cells. Infect Immun 74:6920-6928

27. Wang S, Wang J, Mou H, Luo B, Jiang X (2015) Inhibition of adhesion of intestinal pathogens (Escherichia coli, Vibrio cholerae, Campylobacter jejuni, and Salmonella Typhimurium) by common oligosaccharides. Foodborne Pathog Dis 12:360-365. https://doi. org/10.1089/fpd.2014.1835

28. Semenyuk EG, Laning ML, Foley J (2014) Spore formation and toxin production in Clostridium difficile biofilms. PLoS One 9: e87757. https://doi.org/10.1371/journal.pone.0087757

29. Creti R, Koch S, Fabretti F et al (2006) Enterococcal colonization of the gastro-intestinal tract: role of biofilm and environmental oligosaccharides. BMC Microbiol 6:60. https://doi.org/10.1186/14712180-6-60

30. Powell LC, Pritchard MF, Ferguson EL (2018) Targeted disruption of the extracellular polymeric network of Pseudomonas aeruginosa biofilms by alginate oligosaccharides. Biofilms and Microbiomes 4: 13. https://doi.org/10.1038/s41522-018-0056-3

31. Stabler RA, He M, Dawson L et al (2009) Comparative genome and phenotypic analysis of Clostridium difficile 027 strains provides insight into the evolution of a hypervirulent bacterium. Genome Biol 10. https://doi.org/10.1186/gb-2009-10-9-r102

32. Aubry A, Hussack G, Chen W et al (2012) Modulation of toxin production by the flagellar regulon in Clostridium difficile. Infect Immun 80:3521-3532. https://doi.org/10.1128/IAI.00224-12

Publisher's note Springer Nature remains neutral with regard to jurisdictional claims in published maps and institutional affiliations. 